\title{
G.W. LEIBNIZ, OBRAS FILOSÓFICAS Y CIENTÍFICAS, VOL. 7: ESCRITOS MATEMÁTICOS, ED. MARISOL DE MORA, EDITORIAL COMARES, GRANADA, 2014-15.
}

\author{
RESEÑA: Bernardino Orio de Miguel \\ Madrid
}

Le toca el turno finalmente al volumen 7A+7B, Escritos Matemáticos, 2014-15, que acaba de salir de las prensas en el momento en que se redacta esta recensión. Con diversos traductores y edición de Mary Sol de Mora, presenta en dos entregas los textos esenciales del inmenso e imaginativo quehacer matemático de Leibniz desde su primera juventud hasta el final de su vida, una febril actividad inventiva que abarca todo el espectro matemático de la época: el cálculo binario, las primeras intuiciones sobre el uso de determinantes en las ecuaciones lineales, el arte combinatorio, la teoría de números, desarrollos en serie, el cálculo infinitesimal, la característica aplicada a la geometría más allá de la analítica cartesiana, la probabilidad, los juegos de azar y la estadística, etc, a lo que me referiré luego con un poco más de detalle. Pero antes conviene hacer algunas breves precisiones.

A diferencia de los grandes matemáticos más o menos coetáneos del filósofo (Galileo, Cavalieri, Vallis, Barrow, Descartes, Fermat, los Gregory, los Bernoulli, Huygens, Newton, etc), Leibniz entendió siempre la matemática como un instrumento, un instrumento autónomo sin duda y autosuficiente en el universo inteligible e, incluso, necesario y aplicable a todo razonamiento ○ Dissertatio - Volume Suplementar, Outubro - 2016 
humano, pero al servicio de una visión orgánica de lo real que trasciende todo cálculo, pues lo real, o sea, lo singular, cada mónada, cada suceso del mundo, a diferencia del infinito ideal del cálculo, es un infinito actual irrepetible en la serie sin limite de los hechos (AA VI 4, p. 1515ss; OFC 2, p. 151ss). La matemática, la geometría - la geometría analítica cartesiana, la mathesis universalis -, una geometría sometida todavía al trazado sensible de figuras en el plano, fue en el siglo XVII el paradigma de toda demostración científica. Y así lo entendió Leibniz desde sus años jóvenes. "Yo veía - le dice a Arnauld en 1671, antes de su viaje a París (1672-76) - que la geometría o filosofía del lugar da acceso a la filosofía del movimiento o cuerpo, y la filosofía del movimiento a la ciencia de la mente" (AA II,1, p. 278). Y al duque Johann Friedrich por las mismas fechas: "Tengo intención de escribir unos Elementos acerca de la mente lo mismo que Euclides hizo acerca de la magnitud y la figura, y Hobbes acerca del cuerpo o movimiento" (AA II,1, p. 182). De manera que - añadirá en febrero de 1676, tras el descubrimiento del cálculo diferencial - "sólo la geometría puede proporcionarnos el hilo para salir del laberinto de la composición del continuo y resolver los problemas acerca de máximos y mínimos, acerca de lo inasignable e infinito, de modo que nadie llegará a una sólida metafísica si no ha pasado por la geometría" (AA VI, 3, p. 449). Mas pronto descubrió, precisamente en el cálculo según él, que el continuo funcional siempre interminado de nuestras ecuaciones y de nuestras medidas y requiere para su completa inteligibilidad asumir la existencia de cosas discontinuas, únicas, no extensas, no medibles, verdaderas unidades, que den sentido y realidad a la idealidad de los fenómenos que 
observamos. Resultó así que la geometría - "la geometría más profunda" - se convertía para él en el método dialéctico-platónico de ascenso a lo verdaderamente real, las sustancias simples (ibídem). “Comprendemos así - dirá en 1685 - que unas son las proposiciones que pertenecen a las esencias y otras las que pertenecen a las existencias de las cosas" (AA VI 4, p. 1517; OFC 2, p. 153). Y al final de su vida, pocos meses antes de morir, en un comentario a ciertas críticas que John Toland había hecho a su cálculo y a su sistema de las mónadas, respondía así: “A pesar de mi cálculo infinitesimal, yo no admito un número verdaderamente infinito, aunque confieso que la multitud de las cosas supera todo número finito, incluso todo número (...). El cálculo infinitesimal es útil cuando se trata de aplicar la matemática a la física; y sin embargo, no es mediante él como yo doy cuenta de la naturaleza de las cosas, y considero las cantidades infinitesimales como ficciones útiles" (GP VI, p. 629). Paradójicamente, es precisamente la imperfección actual de las criaturas, frente a la perfección ideal del cálculo, la marca de su singularidad, de su completud interna, de su irrepetibilidad, que se muestra en la materia medible. (GP VII 563s). O si fuera lícito el anacronismo y pudiéramos evocar la actual física de partículas, entenderíamos a Leibniz afirmando, frente al mecanicismo entonces reinante, que el universo es radical y originariamente pura energía existencial, sólo expresable en las infinitas partículas de materia, crecientemente menores sin fin, como los neutrinos u otras partículas que quizás la naturaleza todavía esconde. Y la matemática y demás estrategias del cálculo sería el instrumento imprescindible de acceso a ella, pero corremos el riesgo de quedarnos en el 
cálculo cuando lo que buscamos es lo real, "como les ocurre a los 'materiales', que confunden las condiciones y los instrumentos con la causa verdadera" (GP III, p. 55; GM VI, p. 134; OFC 8, p. 223). “Quien aprehendiera absolutamente tan sólo una única parte de la materia - le decía a Des Bosses en 1710 -, ese tal comprendería absolutamente el universo entero" (GP II p. 412; OFC 14, p. 327).

Sirva, quizás, esta precipitada síntesis para sugerir el lugar que la matemática ocupó siempre en la cosmovisión de Leibniz. Cuando el año 1702 los matemáticos franceses - salvo el Marqués de L’Hôpital - ponían en duda y con razón - el rigor lógico de los infinitésimos leibnizianos, el filósofo tuvo que tranquilizar a su corresponsal Varignon con estas palabras: "No es necesario hacer depender el análisis matemático de las controversias metafísicas ni afirmar que en rigor haya en la naturaleza líneas infinitamente pequeñas en comparación con las nuestras; nos basta explicar lo infinito por lo incomparable, es decir, concebir cantidades más grandes o más pequeñas que las nuestras, de modo que nos contentemos con grados de incomparabilidad" (GM IV, p. 91) y admitamos pragmáticamente la ley de los homogéneos: la suma ; y también el producto $=x d y+y d x+[d x d y]=x d y+y d x$. etc. pues los infinitésimos no son magnitudes reales sino relaciones ficticias de cocientes que se conservan constantes en su creciente pequeñez y son por ello "prescindibles": aquello que es incomparablemente más pequeño es irrelevante introducirlo en el mismo cómputo con aquello que es incomparablemente más grande y hacer en la práctica la operación conmensurable; de esta manera, el error de cálculo será siempre "menor que cualquier error dado", o sea, error nulo (Leibniz en Studia Leibniziana, 
Sonderheft 14, 1986, p. 97-102). Así que pueden y deben los matemáticos "en su oficio" seguir investigando. Y así lo hizo Leibniz en los maravillosos textos que contienen los dos tomos de este volumen "cuando oficiaba de matemático". Aunque Leibniz no fue el primer "inventor" del cálculo de base dos o Cálculo Binario, fue sin duda, tras conocer el I Chin por cartas de los Jesuitas en China, el principal impulsor en Occidente de lo que más tarde sería el Algebra de Boole, como base lógica de los circuitos electrónicos y de la fantástica aplicación a lo que ahora llamamos universo on line. Pues conviene recordar que el Cálculo Binario, lo mismo que el cálculo infinitesimal, eran, para él, sólo pequeñas aplicaciones de aquel magno proyecto de una Analítica y una Combinatoria Universal como instrumentos de la Ciencia General. Ya en 1675, cuando andaba enredado con el nuevo algoritmo, vaticinaba lo siguiente: “A medida que vaya progresando poco a poco el género humano, podrá ocurrir al cabo de muchos siglos que nadie merecerá ya alabanza por la exactitud de su juicio; pues, universalizado el arte analítico, que ahora apenas si se usa sólo de forma correcta y general en las matemáticas, y extendido a toda clase de materias con la ayuda de caracteres filosóficos, tal como yo pretendo, ocurrirá que, dado un tiempo suficiente para la meditación, razonar rectamente no será más meritorio que calcular secuencialmente grandes números" (AA VI 3, p. 429). [Textos I, i-1, i-2].

Siempre insatisfecho con la imperfección de la geometría analítica cartesiana, Leibniz ensayó, por una parte, la sustitución de las letras por números combinatorios en sistemas de dos o tres ecuaciones lineales, a fin de eliminar 
más fácilmente las incógnitas y dar unidad aritmética al sistema; fue ésta una primera intuición, todavía confusa y poco elaborada, de lo que más tarde serían los determinantes de las ecuaciones [Introducción, p. XVII-XX, y Textos II, ii-1 ii-10]. Por otra parte, y a fin de evitar igualmente el "extensionalismo físico y los rodeos inútiles" que las ecuaciones algebraicas requerían, Leibniz pensó una vez más en su combinatoria para construir una nueva geometría abstracta que definiera numéricamente los lugares y sus relaciones, sus semejanzas, los puntos, los espacios, etc, a lo que llamó Característica Geométrica o Analysis situs. De esta manera, podría describirse cualquier objeto en el espacio "sin emplear figuras ni palabras, sino números". A lo largo de toda su vida fue ésta quizás una de sus principales obsesiones matemáticas, que la editora de este volumen nos ofrece en su introducción [p. XXVIII-XXXIV] y en una amplia muestra de textos imprescindibles [Textos IV]. He aquí, una vez más, a Leibniz intuyendo lo que más tarde en el siglo XX sería, ya perfeccionada, la topología. Y no han faltado agudos intérpretes del filósofo que han visto en esta teoría abstracta del espacio uno de los fundamentos científicos de su metafísica, como se mostró al fin en la disputa con Clarke-Newton.

Dada su transcendencia científica e histórica, un capítulo especial merece el descubrimiento del cálculo infinitesimal, al que ya hemos hecho referencia más atrás a propósito de la cosmovisión de Leibniz. Este volumen recoge, entre otras muchas, varias piezas importantes, complicadas sin duda pero fundamentales, que aún no se conocían en versión española. El largo texto de la Cuadratura aritmética del círculo, de la elipse y de la bipérbola, del año 1675/76, 
donde el filósofo expone de manera sistemática su nuevo descubrimiento; y las cuatro cartas, la Epistola Prior y la Epistola Posterior de Newton con sus correspondientes respuestas de Leibniz, de las mismas fechas que la Cuadratura: todo un festín para amantes de la historia del cálculo; y para los menos valientes, el resto de los textos [Textos III. iii-1 - iii-15].

Desde los babilonios y los pitagóricos, la teoría de números y sus fascinantes aplicaciones habían subyugado a los matemáticos de todos los tiempos. El filósofo de Hannover no podía ser una excepción, como él mismo cuenta en numerosas ocasiones [Textos, V]. Fue precisamente en la contemplación de los números primos en su proyecto combinatorio donde Leibniz experimentó por primera vez en su adolescencia la manera de ampliar la lógica aristotélica de proposiciones a los términos simples del conocimiento y redactó su juvenil Dissertatio de arte combinatoria, que se traduce aquí íntegramente [Textos, VI]. Y fue también en el estudio de los números triangulares que, según él, Pascal no había explotado, donde se inició su camino hacia el cálculo infinitesimal [Textos III, iii-14]. A su vez, el estudio de las cónicas, desde Apolonio y Arquímedes, constituyó así mismo una de las fuentes más importantes para la solución de las cuadraturas, que Leibniz resolvió en su análisis. Etc.

Finalmente, la editora nos obsequia en la segunda entrega, volumen 7B (Textos VII-IX), con una serie de textos, si bien menos conocidos no por ello menos importantes, acerca de la enorme variedad de intereses matemáticoprácticos de Leibniz en torno a juegos de azar, análisis de la probabilidad, 
estadística y Seguros, materias todas ellas en las que Mary Sol de Mora es una de nuestras más excelentes expertas (Introducción, p. XXXIX-LXI).

La publicación de este volumen de Escritos Matemáticos de Leibniz debería constituir un hito en la historiografía española acerca de uno de los talentos más geniales y visionarios de nuestra cultura.

Durante los próximos años irán apareciendo otros volúmenes sobre Filosofía del Conocimiento-la Ciencia General-la Enciclopedia, Escritos Teológicos y Religiosos, Escritos Médico-Filosóficos, Escritos Éticos y Politicos, los Nuevos Ensayos, las Correspondencias II, IV, V, VI, para terminar en el último volumen con los Índices de toda la serie de Obras Filosóficas y Científicas de Leibniz. 